\title{
Percepção de políticas de gestão de pessoas e comprometimento organizacional: o papel mediador da percepção de justiça organizacional
}

\author{
Perception of human resources management policies and organizational commitment: the mediating \\ role of perception of organizational justice
}

\section{Renata Gomes de Jesus}

Instituto Federal do Espírito Santo, Campus Guarapari, Coordenadoria de Eletrotécnica, Estrada da Tartaruga, s/n, Muquiçaba, Guarapari, Espírito Santo. 29.215-090, Brasil, renatagj@gmail.com

\section{Diva Ester Okazaki Rowe}

Universidade Federal da Bahia, Núcleo de Pós-graduação em Administração, Avenida Reitor Miguel Calmon, s/n, Vale do Canela, Salvador, Bahia, 40110-903, Brasil, diva@ufba.br

\section{Resumo}

Este artigo teve como objetivo analisar as possíveis influências da percepção de políticas de gestão de pessoas no comprometimento organizacional, verificando o papel mediador da percepção de justiça organizacional. Para tanto, foram utilizadas escalas previamente validadas no contexto brasileiro, a saber: Escala de Políticas de Recursos Humanos, Escala de Percepções de Justiça dos Professores do Ensino Superior, dimensões afetiva, normativa e instrumental da escala de Comprometimento Organizacional. 0 survey on line foi aplicado entre docentes de uma instituição de ensino federal brasileira, que obteve uma amostra de 415 participantes. Para análise dos dados utilizou-se análises multivariadas e modelagens de equações estruturais. Os resultados mostram que as dimensões do comprometimento organizacional são influenciadas pela percepção das práticas de gestão de pessoas "Envolvimento" e "Remuneração" sendo estas influências mediadas pela percepção de justiça. Conclui-se que a percepção positiva de justiça nas práticas de gestão de remuneração e de envolvimento aumenta o comprometimento organizacional, com maior peso nas bases afetiva e normativa.

Palavras-chave: Comprometimento organizacional, políticas de gestão de pessoas, justiça organizacional, docente, instituto federal de ensino.

\section{Abstract}

This article analyzes the possible influences of the perception of political human resource in the organizational commitment, verifying the mediating role of the perception of organizational justice. For this, validated scales in the Brazilian context were used: Human Resources Management Policies and Practices Scale, Perceptions of Justice of Teachers in Higher Education Scale. Afective, Normative and Instrumental dimensions of Organizational Commitment Scale.The survey was administered among teachers in a Brazilian federal institution of education and a sample of 415 teachers was obtained. Multivariate analyzes and structural equation modeling was used. The results show that the dimensions of organizational commitment are influenced by the perception of the practices of human resource "Involvement" and "Remuneration" and these influences are mediated by perception of justice. It's concluded that the positive perception of justice in practices of remuneration management and involvement increases organizational commitment, with greater weight in affective and normative bases.

Key-words: Organizational commitment, human resources management policies, organizational justice, teacher, federal institute of education.

\section{Introdução}

O comprometimento organizacional é o vínculo mais estudado no Brasil pelos pesquisadores do campo de Comportamento Organizacional (Bastos, Maia, Rodrigues, Macambira \& Borges-Andrade, 2014). A pesquisa sobre o tema foi impulsionada no final da década de 1970 e até a década de 1990, o principal modelo para estudos sobre o tema era o proposto por Mowday, Porter e Steers (1982). A partir desta data, o modelo tridimensional proposto por Meyer e Allen (1991) se tornou referência nas pesquisas sobre comprometimento organizacional.

A relevância dos estudos sobre comprometimento organizacional se dá pelo fato de que o comprometimento pode ser visto como o que une os indivíduos em torno do objetivo organizacional e de que este também é um fator potencializador de oportunidades de as organizações obterem sucesso em momentos de instabilidade no mundo corporativo. Ou seja, o comprometimento organizacional e por esta razão deveria ser visto como um importante alvo para os gestores, principalmente os que se voltam para a gestão de pessoas (Brito \& Bastos, 2001).
Bastos, Rodrigues, Moscon, Silva e Pinho (2013) apontam a necessidade da implementação de um conjunto de práticas de gestão que desenvolvam o comprometimento dos trabalhadores com a organização, mas como apontado por Kinnie, Hutchinson, Purcell, Rayton e Swart (2005), o desenvolvimento do comprometimento organizacional dos trabalhadores de uma organização não dependem somente de como são projetadas as políticas de gestão de pessoas, mas de como os empregados percebem e experimentam essas políticas e práticas, o que pode levar a um sentimento de inequidade, que aparece quando o indivíduo percebe que as recompensas não foram proporcionais aos investimentos feitos.

O conceito de percepção de justiça organizacional diz respeito à percepção de como as decisões são tomadas com relação à distribuição de resultados e da justiça percebida em relação aos resultados e é considerada como um dos antecedentes do comprometimento organizacional (Rego, 2003; Rego \& Souto, 2004; Filenga \& Siqueira, 2006; Jamaludin, 2009), ou seja, uma de suas causas.

Na revisão de literatura foram identificadas lacunas de estudos, destacando-se as seguintes. Bastos et al. (2013) 
ressaltam a necessidade de mais estudos entre práticas de gestão de pessoas e comprometimento organizacional. Rowe, Bastos e Pinho (2013), em análise realizada em publicações brasileiras e estrangeiras, apontam reduzida produção científica sobre comprometimento no trabalho entre docentes, indicando a necessidade de mais estudos nesta categoria profissional. Custódio, Siqueira, Viegas, Pinto e Silva (2013) ressaltam que as pesquisas sobre comprometimento no setor público ainda são consideradas incipientes, o que evidencia a demanda por novos estudos. A carência de estudos de gestão de pessoas no setor público brasileiro é apontada por diversos autores. Nesse contexto, delineou-se o seguinte objetivo de pesquisa - analisar a influência da percepção de políticas de gestão de pessoas no comprometimento organizacional, tendo como variável mediadora a percepção da justiça organizacional, em uma amostra formada por docentes de uma instituição de ensino pública federal do Brasil.

Este artigo se encontra organizado da seguinte forma. Seguir-se-á a este capítulo introdutório a exposição do marco teórico sobre os temas abordados e desenvolvimento da hipótese da pesquisa. Na sequência serão descritos os procedimentos metodológicos, os resultados e discussão e as conclusões e recomendações.

\section{Referencial Teórico e Desenvolvimento da Hipótese}

Neste capítulo são apresentados os principais conceitos teóricos relacionados à pesquisa seguidos do desenvolvimento da hipótese deste estudo.

\subsection{Comprometimento organizacional}

Comprometimento Organizacional é definido por Meyer \& Allen (1991) como o estado psicológico caracterizado pela relação entre empregado e organização e que influencia em suas decisões de permanecer ou não como um dos membros desta organização.

A tentativa de compreender o que gera o comprometimento organizacional (antecedentes) e quais são os seus impactos (consequentes) tem sido objetivo de diversas pesquisas, pois sem a compreensão desses aspectos torna-se impossível o seu gerenciamento (Bastos et al., 2013).

Meyer, Stanley, Herscovitch \& Topolnytsky (2002) conduziram uma meta-análise com o objetivo de determinar as relações entre os comprometimentos organizacionais afetivo, instrumental e normativo e as relações entre as variáveis identificadas como seus antecedentes, correlatos e consequentes. As variáveis antecedentes foram divididas em quatro grupos: variáveis demográficas, diferenças individuais, experiências de trabalho e alternativas/investimentos.

Meyer et al. (2002) constataram que as correlações com variáveis demográficas foram geralmente baixas. Destaca-se que os sinais das correlações para todas variáveis de experiências de trabalho envolvendo comprometimento de continuação foram opostos aos dos comprometimentos afetivo e normativo, além de se correlacionarem mais fortemente com o comprometimento afetivo. Os resultados confirmaram um efeito moderado e positivo da percepção da justiça organizacional sobre o comprometimento organizacional afetivo e normativo. A correlação entre a dimensão interacional da justiça e o comprometimento afetivo foi de 0,5 e de 0,52 com o comprometimento normativo. A dimensão distributiva (justiça organizacional) apresentou $\rho=0,40$ e $\rho=0,31$ e a dimensão de procedimentos (justiça organizacional) $\rho=0,38$ e $\rho=0,31$, respectivamente. Sobre o comprometimento de continuação o efeito foi fraco e negativo $(-0,15,-0,06$ e $-0,14$, respectivamente).

Meyer et al. (2002) constataram que as correlações com variáveis demográficas foram geralmente baixas. Destaca-se que os sinais das correlações para todas variáveis de experiências de trabalho envolvendo comprometimento de continuação foram opostos aos dos comprometimentos afetivo e normativo, além de se correlacionarem mais fortemente com o comprometimento afetivo. Como esperado pelos autores, a disponibilidade de alternativas se correlacionou mais fortemente com o comprometimento de continuação do que com os comprometimentos afetivo e/ou normativo.

Morrow (2011) realizou revisão de estudos que aplicaram pesquisa do tipo longitudinal que rendeu seis grandes categorias de antecedentes que têm efeitos sobre o comprometimento afetivo empiricamente demonstrados: práticas de socialização, mudanças organizacionais, práticas de gestão de pessoas, relações interpessoais, as relações empregado-organização e uma categoria residual denominada por ela de "Outros".

Morrow (2011) indica que táticas de socialização institucionalizadas se relacionam positivamente com o comprometimento organizacional e as relações são mais fortes em estudos transversais quando comparadas com aquelas de estudos longitudinais destacando a importância dos fatores de seleção. A pesquisa apontou também que algumas mudanças organizacionais que foram implementadas com o intuito de serem vantajosos para os trabalhadores falharam em produzir resultados positivos. Mudanças como aquisições e downsizing tiveram repercussão negativa e as reações favoráveis à mudança parecem depender de se os funcionários perceberam que seus trabalhos alterados permitiram mais controle pessoal, interdependência de tarefas e recompensas. Sem essas mudanças nas percepções, o nível de comprometimento afetivo pareceu relativamente inalterado.

No que condiz a práticas de gestão de pessoas, Morrow (2011) ressalta que a variável por si só tem efeito limitado no comprometimento afetivo e que a informação e participação em práticas de gestão de pessoas (como conhecimento sobre o desempenho no sistema de avaliação, envolvimento em uma mudança do sistema de pagamentos) que é fundamental para elevar níveis de comprometimento.

Supervisores abusivos e um clima organizacional ruim podem levar à redução do comprometimento afetivo, mas determinados comportamentos do líder como visão inspiradora e esperança podem levar a resultados entre os funcionários que, por sua vez invocam níveis mais elevados de comprometimento afetivo. Além disso, orientação e percepção de comportamentos de cidadania organizacional dos colegas de trabalho oferecem mecanismos potenciais para construção do comprometimento afetivo (Morrow, 2011). 
A classe de antecedentes referente a relações empregadoorganização apresentou evidências fortes de como o comprometimento afetivo pode ser ativamente gerenciado. Para Morrow (2011, p. 29), "incentivar os gestores a focar as percepções dos funcionários sobre a organização empregadora [...] enfatiza a importância de gerar uma parceria com os funcionários onde as preferências do empregado guiam as práticas de gerenciamento".

Não foi encontrada evidência longitudinal que comprovasse que a satisfação no trabalho seja um antecedente do comprometimento organizacional, que a intenção de deixar a organização se trata de um forte preditor do comprometimento organizacional afetivo de ordem causal inversa e que o comprometimento com a carreira pode ser considerado um de seus antecedentes causais positivos (Morrow, 2011).

\subsection{Percepção de Políticas e Práticas de Gestão de Pessoas e de Justiça Organizacional como antecedentes do Comprometimento Organizacional}

Como apresentado na seção anterior, estudos relacionam a percepção positiva das políticas e práticas de gestão de pessoas ao desenvolvimento do comprometimento organizacional. Para Guest (1987), comprometer os empregados com a organização é um dos objetivos das políticas de gestão de pessoas. Contudo, o impacto das práticas de gestão de pessoas sobre o comprometimento recebeu menos atenção do que deveria na visão de Meyer \& Smith (2000).

Guest (1987) define Gestão de Pessoas como a área que compreende um conjunto de políticas com um dos focos na maximização do comprometimento organizacional. Já Legge (2005) afirma que os recursos humanos devam ser aproveitados de forma mais eficaz por meio de políticas consistentes que promovam o comprometimento.

Em estudos sobre gestão de pessoas, Gilliland e Chan (2001) apontam as áreas de recrutamento, seleção e desenvolvimento de pessoas, avaliação de desempenho, benefícios e compensação como aquelas nas quais têm sido aplicado o conceito de justiça organizacional. A forma como os indivíduos percebem a justiça foi apresentada na metaanálise de Meyer et al. (2002) como um dos antecedentes para o comprometimento organizacional, apresentando, nas três dimensões analisadas, correlação positiva com os comprometimentos afetivo e normativo e correlação negativa com o comprometimento de continuação. Hartman, Yrle \& Galle (1999) consideram que o conceito de justiça é essencial para que se possa compreender o comportamento humano nas organizações.

0 número de constructos relacionados à percepção de justiça organizacional não é uma unanimidade entre os pesquisadores. 0 interesse inicial se deu com as pesquisas sobre a justiça distributiva, aquela que lida com a justiça como resultado ou produto final (Hartman et al., 1999) e, a partir de 1975, considerou-se que apenas a justiça distributiva era insuficiente para explicar o construto e passou-se a abordar um novo tipo de justiça que se refere à forma como as decisões são tomadas nas organizações e como são planejadas as tarefas - a justiça de procedimentos ou procedimental.
Atualmente, os estudiosos adotam modelos bi, tri, tetra e pentadimensionais. Para Jamaludin (2009), são três os constructos relacionados à percepção de justiça organizacional - distributiva, de procedimentos e interacional. Entretanto, alguns estudos retornaram a distinguir a justiça de procedimentos da justiça distributiva o que sugere que a justiça interacional seja um subcomponente da primeira e, por isso, adotam o modelo de duas dimensões - justiça de procedimentos e justiça distributiva (Gilliland \& Chan, 2001).

O modelo de quatro dimensões de justiça é adotado por Rego e Souto (2004) por considerarem este de maior valia psicométrica que o modelo tridimensional. No modelo tetradimensional, a vertente interacional é dividida em vertente interpessoal e vertente informacional, sendo as quatro dimensões da justiça organizacional: de procedimentos, distributiva, interpessoal e informacional.

Rego (2001) e Rego, Cunha e Pinho (2009) verificaram que em algumas profissões os indivíduos podem distinguir a dimensão distributiva da justiça nas facetas recompensas e tarefas e propuseram um modelo com cinco dimensões. Para Rego et al. (2009), quando as fontes de alocação de tarefas são distintas, o indivíduo distingue os componentes recompensas e tarefas da dimensão distributiva da justiça. As cinco dimensões são distributiva de tarefas e de recompensas, de procedimentos, interpessoal e informacional.

Para Hayashi, Abib e Bourscheid (2012) o comprometimento dos trabalhadores é influenciado pelas recompensas associadas aos esforços necessários para que se alcance os objetivos organizacionais, ou seja, há uma relação entre justiça e comprometimento organizacional. Filenga e Siqueira (2006), ao analisarem o impacto da percepção de justiça nas três bases de comprometimento organizacional, concluíram que há impacto da percepção de justiça no comprometimento organizacional, sendo que os principais impactos identificados estão relacionados às vertentes distributiva e procedimental.

Filenga e Siqueira (2006) constataram que cerca de 35\% do comprometimento afetivo dos trabalhadores analisados eram explicados pela justiça de procedimentos e que em torno de $18 \%$ do comprometimento normativo e $15 \%$ do de continuação eram explicados pela justiça distributiva. Esse efeito é condizente com resultado similar encontrado por Rego (2003) que constatou que apenas a justiça interpessoal e procedimental explica o comprometimento entre docentes e com a pesquisa de Silva, Silva, Bueno, Rosalem, Souza e Couto (2012) na qual verificou-se que os docentes do ensino superior da amostra eram sensíveis à justiça organizacional, sendo que os professores afetivamente comprometidos com as instituições de ensino nas quais trabalhavam eram os que apresentavam maior percepção de justiça interpessoal.

Meyer e Smith (2000) encontraram forte e significativa correlação entre comprometimento organizacional afetivo e normativo e justiça organizacional. Essas duas dimensões também se correlacionaram significativamente com todas as medidas de avaliação de gestão de pessoas adotadas na pesquisa (desenvolvimento de carreira, benefícios, 
treinamento e avaliação de desempenho). Os resultados da pesquisa confirmaram sugeriram que a percepção das práticas de gestão de pessoas está relacionada com o comprometimento normativo, sendo essa relação mediada pela percepção de justiça de procedimentos.

No mesmo estudo, Meyer e Smith (2000) revelaram que as relações entre as avaliações das práticas de gestão de pessoas por parte dos funcionários e seu comprometimento afetivo e normativo foram em grande parte mediada pela percepção do suporte organizacional e da justiça processual reforçando alegações de que embora as práticas de gestão de pessoas possam ser importantes ferramentas no estabelecimento e manutenção de comprometimento organizacional, os seus efeitos não são nem diretos nem incondicionais e, além disso, os mecanismos por meio dos quais as práticas de gestão de pessoas influenciam as diversas formas de comprometimento podem ser diferentes (Meyer e Smith, 2000). Mais recentemente, Marescaux, De Winne e Sels (2013) argumentam que a diferenciação nas políticas e práticas de gestão de pessoas poderia gerar percepções sobre a favorabilidade dessas políticas por meio da comparação social.

De acordo com essa premissa, coloca-se a seguinte hipótese:

H1: A percepção de justiça organizacional medeia a relação entre a percepção das políticas de gestão de pessoas e o comprometimento organizacional.

A Figura 1 apresenta o desenho conceitual simplificado desta pesquisa, demonstrando a possível influência da percepção das políticas e práticas de gestão de pessoas e da percepção de justiça organizacional, como variável mediadora, no comprometimento organizacional.

Figura 1 - Desenho conceitual geral da pesquisa - Influência da percepção das políticas e práticas de gestão de pessoas no comprometimento organizacional

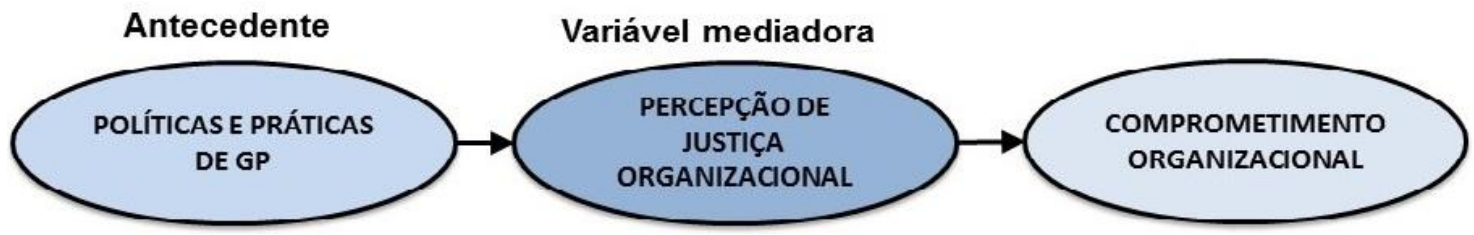

\section{Procedimentos Metodológicos}

O objetivo deste tópico é o de descrever como se deu a coleta, o tratamento e a análise dos dados, bem como descrever a amostra e os instrumentos utilizados.

\subsection{Descrição da amostra e instrumentos}

Foram convidados a participar da pesquisa todos os 1.191 docentes do Instituto Federal do Espírito Santo. A eles foi enviado mensagem de convite por meio de correio eletrônico com um link para acesso ao questionário disponibilizado na ferramenta on line Survey Monkey ${ }^{\circledR}$. Responderam o questionário 415 docentes, após terem concordado com o Termo de Consentimento Livre e Esclarecido. A amostra apresentou média de idade de 28,48 ( $\mathrm{DP}=18,28)$ e média de tempo de serviço na organização de 4,42 anos (DP=8,61), sendo $67,47 \%$ do sexo masculino, $67,47 \%$ casados (as), 48,19\% com mestrado e 77,59\% em regime de trabalho de 40 horas com dedicação exclusiva à organização.

O questionário foi composto com perguntas referentes aos dados pessoais e ocupacionais e constituído das seguintes medidas:

a) Escala de Políticas de Recursos Humanos (EPRH) desenvolvida e validada por Demo, Neiva, Nunes e Rozzett (2012): A percepção dos docentes quanto às políticas de Gestão de Pessoas da organização foi medida por meio de escala composta de 40 itens divididos em seis fatores que representam indicadores ou categorias sobre a variável políticas de gestão de pessoas: envolvimento, treinamento, desenvolvimento e educação, condições de trabalho, recompensas, recrutamento e seleção e avaliação de desempenho e competências, totalizando 40 itens e com formato Likert de cinco pontos. b) Escala de Percepções de Justiça dos Professores do Ensino Superior proposta e validada por Rego (2001) e validada no Brasil por Jesus e Rowe (2014a).

c) Dimensões afetiva e normativa do Comprometimento organizacional: foram usadas as sub-escalas de Meyer \& Allen (1991), composta de seis itens para cada dimensão, com formato Likert de cinco pontos.

d) Dimensão instrumental do Comprometimento Organizacional: foi utilizada a sub-escala de dimensão instrumental revisada por Powell e Meyer (2004) e validada no Brasil por Jesus e Rowe (2014b). Powell e Meyer (2004) recomendam sua adoção em estudos que usassem o modelo de três componentes haja vista que foi constatado que essa versão é mais confiável que a adotada em estudos anteriores. A sub-escala é composta por quatro itens com formato Likert de cinco pontos.

\subsection{Procedimentos de Análise}

Para análise dos dados foram realizadas análises exploratórias (análise e tratamento dos outliers, avaliação da normalidade, linearidade, multicolineariedade e variâncias relativas) e análise da mensuração das escalas (validade convergente e discriminante das medidas). A ferramenta on line utilizada para coleta dos dados desta pesquisa não permitiu missing values. Por esta razão, a primeira análise realizada foi a de outliers, que revelou cinco variáveis com valores extremos univariados e 52 casos de outliers multivariados. Preferiu-se manter todos os valores originais dado a impossibilidade de se excluir os respondentes sem causar uma perda excessiva de dados na amostra, bem como pela constatação de que tais valores representam casos válidos da população. 
Para análise da normalidade foi realizado o teste de Kolmogorov-Smirnov e, conforme esperado, ocorreram desvios da normalidade em basicamente todos os indicadores dos construtos. A fim de investigar a magnitude e as cautelas a serem tomadas quanto ao não atendimento do pressuposto de normalidade da amostra, avançou-se para análise da magnitude dos desvios dos parâmetros de assimetria e curtose. Parte considerável das variáveis apresenta desvios em parâmetros de assimetria fora dos limites aceitáveis. Entretanto, dado o tamanho da amostra, pode-se considerar que ela permite relativa segurança no emprego de métodos tradicionais de equações estruturais que assumem a normalidade (Hair, Black, Babin, Anderson \& Tatham, 2009).

Na sequência foi analisada a dimensionalidade de todas as escalas utilizadas por meio da Análise Fatorial Exploratória pelo Método dos Componentes Principais, aplicando o critério de Screeplot e eigenvalue superior a 1 e método de rotação oblíqua (direct Oblimin), visando obter fatores teoricamente significativos que representam o agrupamento de variáveis com maior precisão.

Finalizada a fase exploratória, foi efetuada a análise fatorial confirmatória por meio de modelagem de equações estruturais utilizando-se o programa AMOS. Em um primeiro momento foi testada a análise fatorial confirmatória para cada sub-escala isolada, para depois testar um modelo completo, considerando todas as dimensões encontradas.

Avançou-se para a avaliação da validade do modelo verificando-se a validade convergente e descriminante. A validade convergente foi analisada pela significância das cargas fatoriais não padronizadas e todos os indicadores apresentaram validade aceitável. Segundo o critério de cargas superiores a 0,5 foi preciso excluir quatro indicadores por apresentarem ausências de validade e cargas menores que 0,5. Todas as demais apresentaram validade aceitável.

Somente dois construtos apresentaram valores de variância partilhada com os indicadores (AVE) inferiores a 0,4, mas por pequeno percentual em relação ao benchmark. A validade discriminante foi analisada por meio da comparação da variância entre os construtos (R2) e AVE, mostrando alguns desvios. Nestes casos empregou-se o teste de diferença qui-quadrado.

O modelo conceitual foi então testado por meio de Modelagem de Equações Estruturais utilizando o software AMOS. Decidiu-se testar o modelo considerando a justiça um fator reflexivo de segunda ordem, dado que o ajuste do modelo fatorial comparando uma estrutura de segunda ordem a um modelo de quatro fatores correlacionados, mostrou melhor qualidade do ajuste para a estrutura com fator de segunda ordem. Ademais, a tentativa de testar o modelo estrutural considerando quatro dimensões de justiça revelou uma solução não apropriada, com presença de variância de erro negativo em vários construtos, o que é forte indício do erro de especificação do modelo (Kline, 2005). Assim, considerando resultados com variâncias negativas do erro de previsão das dimensões de justiça a elevada correlação e índices de ajustes de parcimônia favoráveis ao modelo de segunda ordem, tratou-se a justiça como um fator reflexivo de segunda ordem.

\section{Resultados e discussão}

0 modelo mais parcimonioso obtido está apresentado na Figura 2, onde é possível verificar que as dimensões de gestão de pessoas que explicaram a percepção de justiça foram "Envolvimento" e "Remuneração", com peso muito superior da primeira $(0,876)$ em relação a segunda $(0,194)$. Os dois construtos explicaram $87,8 \%$ da justiça. A justiça (fator de segunda ordem) explicou todas suas dimensões com elevados percentuais, a saber - distributiva de tarefas, distributiva de disciplinas, distributiva de recompensas e interacional. Finalmente, a justiça explicou todas as dimensões de comprometimento, com maior força nas dimensões normativa $(0,689)$ e afetiva $(0,695)$ e, com menor peso, a instrumental $(0,348)$.

Figura 2 - Modelo da pesquisa - Influências da percepção das políticas e práticas de gestão de pessoas no comprometimento organizacional

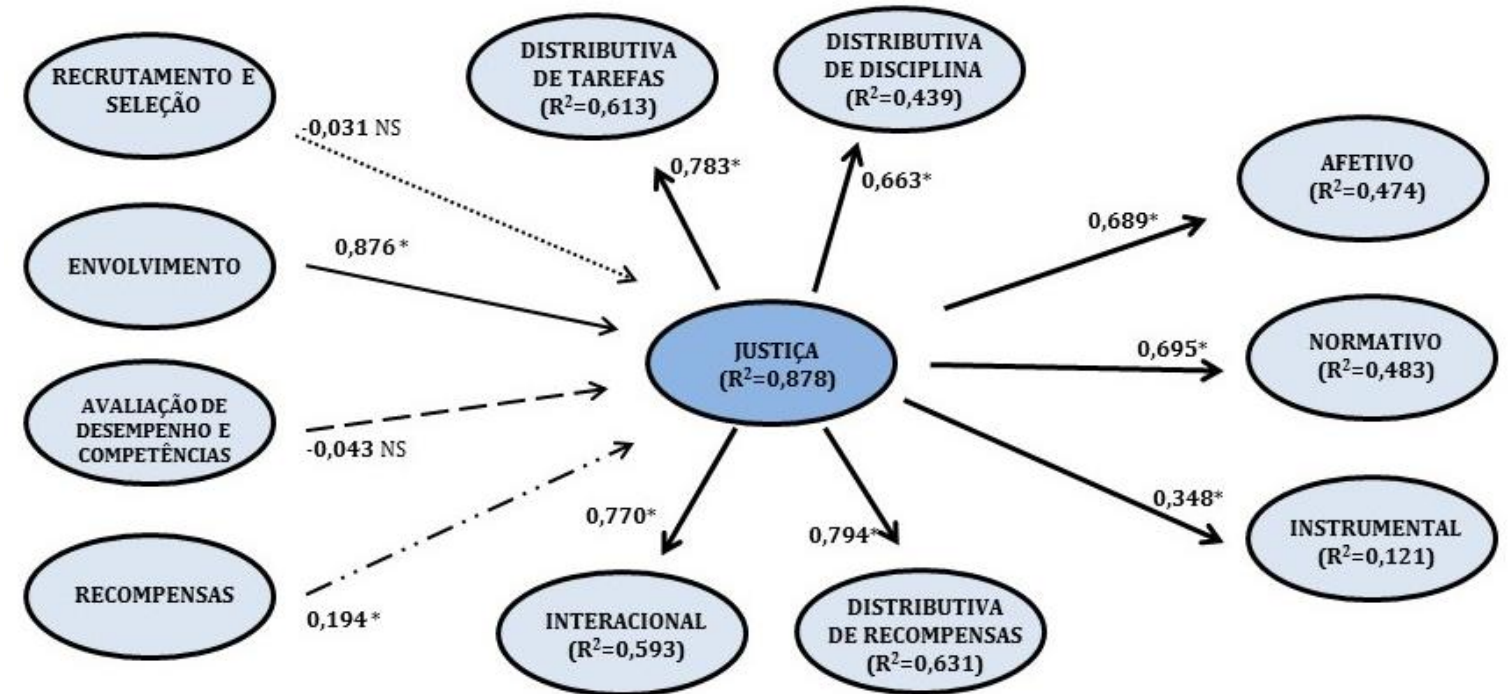

${ }^{*}$ Significativo ao nível de $0,1 \%$ unicaudal $(\mathrm{p}<0,001)$. NS - Não significativo. R² - Percentual de variância explicada.

Fonte: Dados da pesquisa. 
A Tabela 1 mostra os pesos padronizados e não padronizados das relações estruturais. Vale ressaltar que dois fatores da escala de percepção de gestão de pessoas, 'recrutamento e seleção' e 'avaliação de desempenho e competências', não influenciam a percepção de justiça organizacional, entre os participantes desta pesquisa. Pode ser influenciado pelo fato de que recrutamento e seleção de docentes no setor público brasileiro é realizado por meio de concurso público, atendendo toda legislação específica.

Tabela 1 - Pesos padronizados e não padronizados das relações estruturais

\begin{tabular}{|c|c|c|c|c|c|c|c|}
\hline $\begin{array}{c}\text { Construtos } \\
\text { independentes }\end{array}$ & & Construtos dependentes & $\begin{array}{l}\text { Peso } \\
\text { padrão }\end{array}$ & $\begin{array}{c}\text { Peso } \\
\text { regressão }\end{array}$ & $\begin{array}{l}\text { Erro } \\
\text { padrão }\end{array}$ & Valor $\mathrm{t}$ & Sig. \\
\hline Recrutamento e Seleção & $\rightarrow$ & \multirow{4}{*}{ Justiça $\left(\mathrm{R}^{2}=0,878\right)$} & $-0,031$ & $-0,057$ & 0,16 & $-0,355$ & 0,723 \\
\hline Remuneração & $\rightarrow$ & & 0,194 & 0,201 & 0,051 & 3,924 & $<0,001$ \\
\hline $\begin{array}{l}\text { Avaliação de desempenho } \\
\text { e competências }\end{array}$ & $\rightarrow$ & & $-0,043$ & $-0,055$ & 0,124 & $-0,441$ & 0,659 \\
\hline Envolvimento & $\rightarrow$ & & 0,876 & 1,175 & 0,186 & 6,309 & $<0,001$ \\
\hline \multirow{4}{*}{ Justiça } & $\rightarrow$ & $\begin{array}{l}\text { Distributiva de } \\
\text { Recompensas }\left(\mathrm{R}^{2}=0,631\right)\end{array}$ & 0,794 & 1 & --- & --- & --- \\
\hline & $\rightarrow$ & $\begin{array}{l}\text { Interacional } \\
\left(\mathrm{R}^{2}=0,593\right)\end{array}$ & 0,770 & 0,857 & 0,066 & 12,979 & $<0,001$ \\
\hline & $\rightarrow$ & $\begin{array}{l}\text { Distributiva de Disciplina } \\
\left(\mathrm{R}^{2}=0,439\right)\end{array}$ & 0,663 & 0,874 & 0,078 & 11,268 & $<0,001$ \\
\hline & $\rightarrow$ & $\begin{array}{l}\text { Distributiva de Tarefas } \\
\left(\mathrm{R}^{2}=0,613\right)\end{array}$ & 0,783 & 0,77 & 0,062 & 12,374 & $<0,001$ \\
\hline Justiça & $\rightarrow$ & Afetivo $\left(\mathrm{R}^{2}=0,474\right)$ & 0,689 & 0,495 & 0,048 & 10,256 & $<0,001$ \\
\hline Justiça & $\rightarrow$ & Normativo $\left(R^{2}=0,483\right)$ & 0,695 & 0,501 & 0,057 & 8,794 & $<0,001$ \\
\hline Justiça & $\rightarrow$ & Instrumental $\left(\mathrm{R}^{2}=0,121\right)$ & 0,348 & 0,285 & 0,049 & 5,765 & $<0,001$ \\
\hline
\end{tabular}

Os índices de ajuste do modelo estão próximos aos patamares aceitos, apresentando GFI com valor igual a 0,821 e CFI igual a 0,882 (o valor mínimo sugerido pela literatura é de 0,9). Estes valores são explicados pelo grande tamanho do modelo. Por outro lado, o RMSEA atingiu nível satisfatório, ficando inferior a 0,08 $(0,061)$ e o índice $\chi^{2} /$ g.l. foi de 2,523 .

Para realizar os testes de mediação empregou-se o método de comparação do valor qui-quadrado do modelo em que o peso estrutural entre a variável independente (VI) e a variável dependente (VD) é estimado livremente (modelo irrestrito), com o qui-quadrado do modelo (aninhado) em que o peso da relação direta $(\mathrm{VI} \rightarrow \mathrm{VD})$ é fixo em zero $(0)$ (modelo restrito) (VIEIRA, 2009).

Quando a diferença qui-quadrado entre os modelos é inferior a 3,814, pode-se dizer, com 95\% de confiança, que o efeito direto não é significativo, então não existe relação direta (VI $\rightarrow$ VD) e ocorre a mediação total. No entanto, para que isso ocorra é necessário que; 1) a relação entre a variável independente (VI) e a dependente (VD) seja significativa, quando a relação entre a mediadora (Med) e a variável dependente (VD) é fixa em zero (0); 2) a relação entre a variável independente (VI) e a mediadora (Med) seja significativa, quando o efeito direto é nulo $(\mathrm{VI} \rightarrow \mathrm{VD}=0)$ e; 3) a relação entre a variável mediadora (Med) e a dependente (VD) seja significativa quando o efeito direto é nulo $(\mathrm{VI} \rightarrow \mathrm{VD}=0)$.

Quando a diferença qui-quadrado entre os modelos supera o valor de 3,814, pode-se dizer, com $95 \%$ de confiança, que o efeito direto é significativo, pois a relação direta (VI $\rightarrow$ VD) é suportada. Neste caso podem ocorrer duas situações. $\mathrm{Na}$ primeira, se o efeito da variável independente (VI) sobre o mediador (Med) é significativo, conjugando ao efeito significativo entre o mediador (Med) e a variável dependente (VD), tem-se a mediação parcial, pois parte do efeito de VI sobre a VD será repassado através da Med. No segundo caso, se o efeito direto da Mediadora (Med) sobre a variável dependente passar a ser não significativo, não haverá mediação . Na Tabela 2, demonstra-se a relação da diferença qui-quadrado do modelo restrito menos o valor do modelo irrestrito.

Como todos os modelos irrestritos não foram significativamente diferentes dos modelos restritos, chegase a conclusão que todos os efeitos diretos, na presença da variável moderadora, são iguais a 0 . Assim, havendo mediação ela terá que ser total. Efeito da variável independente sobre a mediadora e da mediadora sobre a variável dependente, critério que deve ser verificado para confirmar a mediação, só ocorreu para a relação das variáveis Envolvimento e Recompensas. Para configurar a mediação, testaram-se modelos em que o peso das mediadoras sobre as variáveis dependentes foi fixo em zero (0) e estimou-se o efeito direto da variável independente sobre a variável dependente. Caso esta relação não seja significativa não há o que se falar em mediação.

Para o Envolvimento essa relação foi significativa para o comprometimento afetivo ( $b=0,652 ; t=9,547 ; p<0,001)$, normativo $(b=0,639 ; t=8,362 ; p<0,001)$ e instrumental $(b=0,290 ; t=4,880 ; p<0,001)$. Assim configura-se que, na ausência da relação entre justiça e os três tipos de comprometimento, existe uma relação direta significativa entre o envolvimento, mas que, considerando o elemento justiça como mediador, esta relação direta deixa de existir, sendo totalmente mediada pelo grau de justiça percebida.

Para Recompensas essa relação foi significativa para o comprometimento afetivo $(b=0,473 ; t=6,922 ; p<0,001)$, 
normativo $(b=0,552 ; t=7,540 ; p<0,001)$ e instrumental $(b=0,310 ; t=4,975 ; p<0,001)$. Mais uma vez pode-se atestar a mediação total, pois na ausência da relação entre justiça e os três tipos de comprometimento, existe uma relação direta significativa entre a remuneração. No entanto esta relação desaparece à medida que a justiça entra como mediadora.

Esses dados revelam que quanto mais o professor percebe de forma positiva as práticas gestão de pessoas relacionadas a "Envolvimento" e a "Recompensas" e percebe também de forma positiva a justiça organizacional, aumenta-se a probabilidade de que se desenvolva o comprometimento organizacional em suas três dimensões.

\section{Conclusões e Recomendações}

O objetivo deste artigo foi analisar a influência da percepção de políticas de gestão de pessoas no comprometimento organizacional, tendo como variável mediadora a percepção da justiça organizacional, haja vista que estudos anteriores (Meyer \& Smith, 2000) revelaram que a relação entre as avaliações das práticas de gestão de pessoas por parte dos empregadose seu comprometimento afetivo e normativo era mediada pela percepção da justiça processual. Como a amostra é constituída por docentes, optou-se por adotar o modelo pentadimensional proposto por Rego (2001) já que este modelo considera que alguns profissionais distinguem a dimensão distributiva em duas vertentes - recompensas e tarefas.

Os resultados confirmaram a hipótese da pesquisa, demostrando que as três dimensões do comprometimento organizacional são significativamente influenciadas pela percepção das práticas de gestão de pessoas "Envolvimento" e "Recompensas" sendo esta relação totalmente mediada pela percepção de justiça. Resultado similar foi verificado por Demo, Martins \& Roure (2013) que encontraram como melhores preditoras do comprometimento organizacional as políticas de envolvimento e as políticas de recompensas e por Del Corso, Petraski, Silva e Taffarel (2014) que constataram que o envolvimento aumenta o comprometimento com os objetivos da organização, pois as pessoas passam a ser responsáveis pelos indicadores.

Bastos et al. (2013) ressaltam que das práticas de gestão de pessoas, a que menos recebe atenção dos pesquisadores no que tange a sua influência sobre o comprometimento organizacional é a de administração de salários e benefícios e, fundamentados nas bases teóricas do comprometimento, afirmam que a possibilidade de impacto positivo no comprometimento do trabalhado está atrelada ao suporte organizacional representado pelos benefícios e políticas de remuneração, mas sinalizam que se isolado de outras práticas de gestão de pessoas que promovam o comprometimento o vínculo gerado pode ser mais instrumental.

Entretanto, neste trabalho foi encontrado que as principais influências da percepção de práticas de gestão de pessoas estão relacionadas às dimensões afetiva e normativa do comprometimento organizacional. Desta forma, os resultados sugerem que uma percepção positiva de justiça nas práticas de remuneração pode influenciar de forma positiva o comprometimento dos docentes, desenvolvendo neles o desejo (comprometimento afetivo) e a obrigação (comprometimento normativo) de permanecerem na organização. Vale ressaltar que ambas dimensões se relacionam positivamente com consequentes desejados como desempenho e cidadania organizacional enquanto que a dimensão instrumental se relaciona negativamente (Meyer et al., 2002).

No que diz respeito à relação entre percepção de práticas de gestão de pessoas e justiça a maior relação estrutural encontrada foi a do construto envolvimento. Como principal preditora da percepção da justiça organizacional, tal prática deve receber atenção especial por parte dos gestores de pessoas, visto que aspectos relacionados ao envolvimento do funcionário, como comunicação e participação, já foram associados positivamente ao desenvolvimento do comprometimento (Mathieu \& Zajac, 1990; Bandeira, Marques \& Veiga, 2000).

Como limitação deste estudo, ressalta-se que foi necessária a adoção do fator de segunda ordem no construto "Justiça organizacional" o que impossibilitou o teste de relações entre os fatores de primeira ordem e as dimensões do comprometimento organizacional de forma a verificar se todas as dimensões da justiça organizacional influenciam as dimensões do comprometimento organizacional da mesma forma (Hair et al., 2009).

É interessante ressaltar que a amostra utilizada nesta pesquisa foi formada por uma única categoria profissional, docentes, de um Instituto Federal de Educação, Ciência e Tecnologia, instituição de ensino pública na qual o docente atua do ensino médio à pós-graduação. Por esta razão, indica-se a reaplicação desta pesquisa ampliando a amostra de docentes para outras instituições que façam uso de políticas e práticas de gestão de pessoas distintas, como Universidades públicas e privadas de forma a verificar se há diferença entre diferentes trabalhadores.

Além disso, considerando-se a necessidade de estudos que possibilitem examinar as mudanças nos níveis de comprometimento organizacional ao longo do tempo apontada por diversos pesquisadores do comprometimento organizacional (Mowday et al.,1982; Morrow, 2011), uma pesquisa futura poderia verificar o papel mediador da percepção de justiça organizacional adotando uma perspectiva longitudinal.

\section{Agradecimentos}

Agradecemos ao Conselho Nacional de Desenvolvimento Científico e Tecnológico (CNPq) pelo apoio financeiro a essa pesquisa.

\section{Referências}

Bandeira, M., Marques, A. \& Veiga, R. (2000). As dimensões múltiplas do comprometimento organizacional: um estudo na ECT/MG. Revista de Administração Contemporânea, 4(2), 133-157.

Bastos, A., Rodrigues, A., Moscon, D., Silva, E. \& Pinho, A. (2013). Comprometimento no trabalho: fundamentos para a gestão de pessoas. In: L. Borges \& L. Mourão (Org.). $O$ trabalho $e$ as organizações: atuações a partir da Psicologia 1ํㅡㄹ ed. (pp. 279-310). Porto Alegre: Artmed. 
Bastos, A. V. B., Maia, L. G. M., Rodrigues, A. C. D. A., Macambira, M. 0., \& Borges-Andrade, J. E. (2014). Vínculos dos indivíduos com a organização: análise da produção científica brasileira 2000-2010. Psicologia: Teoria e Pesquisa, 30(2), 153-162.

Brito, A. \& Bastos, A. (2001). O Schema de "trabalhador comprometido" e gestão do comprometimento: um estudo entre gestores de uma organização petroquímica. Organizações $e$ Sociedade, 8(22), 177-193.

Custódio, L., Siqueira, C., Viegas, G., Pinto, V. \& Silva, R. (2013). Comprometimento organizacional e estratégias de gestão de pessoas: uma análise das experiências em organizações públicas brasileiras. Anais dos Seminários em Administração - SemeAd, São Paulo, SP, Brasil, 16.

Del Corso, J. M., Petraski, S. J., Silva, W. V. D., \& Taffarel, M. (2014). Gestão estratégica de recursos humanos: identificando o processo de alinhamento estratégico. Tourism \& Management Studies, 10(ESPECIAL), 49-57.

Demo, G., Neiva, E., Nunes, I. \& Rozzett, K. (2012). Human resources management policies and practices scale (HRMPPS): Exploratory and confirmatory factor analysis. BAR Brazilian Administration Review, 9(4), 395-420.

Demo, G., Martins, P. \& Roure, P. (2013). Políticas de gestão de pessoas, comprometimento organizacional e satisfação no trabalho na livraria cultura. Revista Alcance - Eletrônica, 20(2), 237-254.

Filenga, D. \& Siqueira, M. (2006). O impacto de percepções de justiça em três bases de comprometimento organizacional. R. Adm., 41(4), 431-441.

Gilliland, S. \& Chan, D. (2001). Justice in organizations: theory, methods, and applications. In: N. Anderson, D. Ones, H. Sinangil \& C. Viswesvaran, Handbook of industrial, work and organizational psychology (pp. 143-165). Londres: SAGE Publications.

Guest, D. (1987). Human resource management and industrial relations. Journal of Management Studies, 24(5), 503-521.

Hair, I., Black, W., Babin, B., Anderson, R. \& Tatham, R. (2009). Análise multivariada de dados. 6⿳亠丷厂 Ed. Porto Alegre: Bookman..

Hartman, S., Yrle, A. \& Galle, W. (1999). Procedural and distributive justice: examining equity in a university setting. Journal of Business Ethics, (20), 337-351.

Hayashi, P., Abib, G. \& Bourscheid, M. (2012). Transformando os recursos humanos em estratégicos: como cobrar adequadamente as pessoas. In: Book of Proceedings - TMS - Management Studies International Conference Volume 2: ESGHT - Universidade do Algarve, 604-613.

Jamaludin, Z. (2009). Perceived organizational justice and its impact to the development of commitments: a regression analysis. World Journal of Management, 1(1), 49-61.

Jesus, R. \& Rowe, D. (2014a). Justiça organizacional percebida por professores dos ensinos básico, técnico e tecnológico. RAM. Revista de Administração Mackenzie, 15(6), 172-200.

Jesus, R. \& Rowe, D. (2014b). Comprometimento organizacional: tradução, adaptação e validação para o contexto brasileiro da escala de sacrifícios percebidos associados com a saída. Anais do Encontro de Estudos Organizacionais da ANPAD - EnEO, Gramado. RS, Brasil, 8.

Kinnie, N., Hutchinson, S., Purcell, J., Rayton, B. \& Swart, J. (2005) Satisfaction with HR practices and commitment to the organisation: Why one size does not fit all. Human Resource Management Journal, 15(4), 9-29. Kline, R. B. (2011). Principles and Practice of Structural Equation Modeling. Third edition. New York (USA): Guilford Press.

Legge, K. (2005). Human resource management: rhetorics and realities. Anniversary Edition. London: Palgrave Macmillan.

Marescaux, E., De Winne, S., \& Sels, L. (2013). HR practices and affective organisational commitment:(when) does HR differentiation pay off?. Human Resource Management Journal, 23(4), 329-345.

Mathieu, J. \& Zajac, D. (1990). A review and meta-analysis of the antecedents, correlates, and consequences of organizational commitment. Psychological Bulletin, 108(2), 171-194.

Meyer, J. \& Allen, N. (1991). A three-component conceptualization of organizational commitment. Human Resource Management Review, 1(1), 61-89.
Meyer, J. \& Smith, C. (2000). HRM practices and organizational commitment: test of a mediation model. Canadian Journal of Administrative Sciences, 17 (4), 319-331.

Meyer, J., Stanley, J., Herscovitch, L. \& Topolnytsky, L. (2002). Affective, continuance, and normative commitment to the organization: a meta-analysis of antecedents, correlates, and consequences. Journal of Vocational Behavior, 61, 20-52.

Morrow, P. (2011). Managing organizational commitment: insights from longitudinal research. Journal of Vocational Behavior, 79, 18-35.

Mowday, R., Porter, L. \& Steers, R. (1982). Employee-organization linkages: the psychology of commitment, absenteeism, and turnover. New York: Academic Press.

Powell, D. \& Meyer, J. (2004). Side-bet theory and the threecomponent model of organizational commitment. Journal of Vocational Behavior, 65, 157-177.

Rego, A. (2001). Percepções de justiça: estudos de dimensionalização com professores do ensino superior. Psicologia: Teoria e Pesquisa, 17(2), 119-131.

Rego, A. (2003). Climas de justiça e comprometimento organizacional. $r P O T, 3(1), 27-60$.

Rego, A. \& Souto, S. (2004). A percepção de justiça como antecedente do comprometimento organizacional: um estudo LusoBrasileiro. RAC, 8(1), 151-177.

Rego, A., Cunha, M. \& Pinho, C. (2009). Exploring a five-factor model of organizational justice. Management Research, 7(2), 103-125.

Rowe, D., Bastos, A. \& Pinho, A. (2013). Múltiplos comprometimentos com o trabalho e suas influências no desempenho: um estudo entre professores do ensino superior no Brasil. Organizações \& Sociedade, 20(66), 501-521.

Silva, A. V. da, Silva, R. L. F. C., Bueno, M., Rosalem, V., Souza, A. M. de, \& Couto, G. (2012). Comprometimento afetivo organizacional: reflexões sobre o papel das percepções de justiça. Encontro: Revista de Psicologia, 15(23), 73-82.

Vieira, V. A. (2009). Moderação, mediação, moderadora-mediadora e efeitos indiretos em modelagem de equações estruturais: uma aplicação no modelo de desconfirmação de expectativas. Revista de Administração da USP, 44(1), 17-33.

Submetido: 23.06 .2014

Aceite: 25.11.2014 